\title{
Effect of the monomer structure on the dynamics of semidilute polyalkylmethacrylate solutions: A quasielastic light and neutron scattering investigation
}

\author{
S. Magazù ${ }^{\mathrm{a})}$ \\ Dipartimento di Fisica and INFM dell'Università di Messina, C.da Papardo S.ta Sperone 31, \\ 98166 Messina, Italy \\ V. Villari \\ Istituto di Tecniche Spettroscopiche-CNR, Via La Farina 237, 98123 Messina, Italy \\ A. Faraone and $\mathrm{G}$. Maisano \\ Dipartimento di Fisica and INFM dell'Università di Messina, C.da Papardo S.ta Sperone 31, \\ 98166 Messina, Italy \\ S. Janssen \\ Laboratory for Neutron Scattering, Paul Scherrer Institute, CH-5232 Villigen, Switzerland
}

(Received 9 July 2001; accepted 4 October 2001)

\begin{abstract}
The study of the microscopic origin of the slow relaxation modes in the autocorrelation functions of some entangled polymer solutions has recently given rise to much experimental and theoretical work. The aim is to highlight the role of concentration, molecular weight, and temperature on the slow modes and to what extent the entanglement phenomena are responsible for their appearance. The present work reports on the comparison between the dynamics of homologous polymers with different side unit lengths, by use of quasi-elastic light and neutron scattering. The investigation, carried out on both mesoscopic and microscopic scales, with different concentrations and chain lengths, evidenced different dynamical properties in the three polymer solutions. In particular, longer side chains give rise to a bigger volume excluded to the other segments, obstructing the entanglements responsible for the slow relaxation modes and favoring the mobility of segments.

(C) 2002 American Institute of Physics. [DOI: 10.1063/1.1421355]
\end{abstract}

\section{INTRODUCTION}

One of the main goals of polymer science is to relate the macromolecular chain structure to the macroscopic properties. The interplay between the physical properties of a polymer chain and its unperturbed dimension was already enunciated by Flory in 1969. ${ }^{1,2}$ In particular, it has been hoped that one could relate the polymer coil size to the entanglement degree. Entanglements are the origin of the high viscosity of semidilute solutions of large polymers, ${ }^{3}$ and it has been shown both experimentally ${ }^{4-9}$ and theoretically ${ }^{10-15}$ that they may have a strong influence on the dynamics of concentration fluctuations as probed by Dynamic Light Scattering (DLS). Recent studies on some semidilute and moderately concentrated polymer solutions revealed that the dynamic structure factor is multimodal and may be separated into two parts, usually called fast and slow modes. An issue of great interest is the recent debate, both theoretical and experimental, over the theory of Wang, ${ }^{10,15}$ which predicts that the slow mode disappears when polymer and solvent have identical partial specific volumes. The early theory of Brochard and de Gennes, ${ }^{9}$ later improved by Adam and Delsanti, ${ }^{16}$ relates the presence of the slow mode to the nonzero elastic longitudinal modulus of the transient gel constituted by the entangled chains. More recently Wang, using a

${ }^{\text {a)} E l e c t r o n i c ~ m a i l: ~ m a g a z u @ d s m e 01 . u n i m e . i t ~}$ thermodynamic approach based on the Bearman-Kirkwood equation for a binary solution, found quite similar results to those obtained by Adam and Delsanti, but the slow mode is now connected with the elastic longitudinal modulus of the polymer solution. Moreover in Wang's theory the slow mode contribution is weighted by the factor $\beta^{2}$, where $\beta$ $=c \rho^{-1}(\partial \rho / \partial c)_{T, P}(\rho$ being the density and $c$ the concentration of the solution) is a dimensionless densityconcentration coupling parameter. Even if some experimental findings confirm the role played by the $\beta$ parameter, the link between the zero value of $\beta$ and the disappearance of the slow mode contribution is not yet completely clear. ${ }^{17}$

In a previous work ${ }^{18}$ by our group on PMMA/acetone solutions the conformational and dynamical properties of the polymeric chains as a function of concentration, temperature and molecular weight were investigated. The increase of the diffusion coefficient at low concentration and the fact that its extrapolation to infinite dilution obeyed the scaling law $D_{0}$ $=a M_{w}^{-\nu}$, with $\nu=0.57$, indicated a swollen state of the polymer molecules, namely the existence of repulsive interactions between monomers. A more striking feature appeared in the semidilute regime in which entanglements cannot be neglected and influence the overall dynamics of the polymer chains. In fact, the autocorrelation functions of these systems showed a net distinction between a fast relaxation mode, attributed to the collective diffusive process, and a broad 
distribution of slow modes. We hypothesized that, because the slow processes sometimes do not occur in the circumstances in which they are expected, the entanglement effects, which are manifested above a certain minimum concentration and polymer chain length, could also depend on the monomer structure constituting the chain.

The present work, in which we deal with the comparison between the dynamics of polymers belonging to the same family [Poly(Alkyl MethAcrylates), PAMA] and having a different side unit length, must be inserted in this frame. The new experimental results show that the side unit length, affecting the conformational properties of the polymer, can trigger the appearance of the slow relaxation modes.

PAMA are an interesting class of materials employed in applications in nonlinear optics, holographic data handling, and energy storage. ${ }^{19-21}$ A better understanding of their molecular dynamics and, in particular, the influence of the side chain movements, would improve the relevant properties of materials employed in these fields. Although these polymers have been widely investigated in the melt phase and near the glass transition, ${ }^{22-25}$ less attention has been devoted to their properties in solution, in dilute and semidilute regimes, and to what extent side chain motions are relevant in solution.

In the experimental approach two techniques were employed, Quasi-Elastic Light (QELS) and Neutron Scattering (QENS): light scattering allows the investigation of the collective dynamics of the polymers on a micrometric scale and neutron scattering the investigation of the monomer internal dynamics. Moreover, comparing the hydrogenated and partially deuterated spectra, we could determine the components of the experimental spectra originating from the main chain and from the side chain dynamics.

\section{SAMPLES AND EXPERIMENTAL SETUP}

In order to evaluate the role played by the monomer structure on the relaxation properties in semidilute polymer solutions, we investigated the first three members of the Polyalkylmethacrylate series. ${ }^{26-28}$ These homologous polymers are PolyMethylMethAcrylate (PMMA, - $\left[\mathrm{CH}_{2} \mathrm{C}\left(\mathrm{CH}_{3}\right)\right.$ $\left.\left(\mathrm{CO}_{2} \mathrm{CH}_{3}\right)\right]_{n^{-}}$), PolyEthylMethAcrylate (PEMA, - $\left[\mathrm{CH}_{2} \mathrm{C}\right.$ $\left.\left.\left(\mathrm{CH}_{3}\right)\left(\mathrm{CO}_{2} \mathrm{CH}_{2} \mathrm{CH}_{3}\right)\right]_{n}{ }^{-}\right)$and PolyButylMethAcrylate (PBMA, - $\left[\mathrm{CH}_{2} \mathrm{C}\left(\mathrm{CH}_{3}\right)\left(\mathrm{CO}_{2}\left(\mathrm{CH}_{2}\right)_{3} \mathrm{CH}_{3}\right)\right]_{n}$-); they differ from each other in the number of $\mathrm{CH}_{2}$ groups each side unit has. The samples used, furnished by the Aldrich Chemical Company, are highly monodisperse (the nominal polydispersity is 1.1) and have different molecular weights (see Table I).

The solutions were prepared by dissolving a weighted amount of polymer in acetone and were left for a few hours before the measurement in order that they could stabilize. It was also necessary to filter the solutions in recirculation, by means of a PTFE Amicon millipore filter of $0.45 \mu \mathrm{m}$, to minimize the effects due to the presence of dust or impurities. In order to check if and to what extent the filtration procedure altered the composition of the sample, we also prepared, from a solution having an intermediate concentration value, two couvettes. One was filtered and the other centrifuged. The correlation functions obtained for the two samples gave the same result, the discrepancy being less than $2 \%$. However, because of the fact that the filtered sample
TABLE I. $R_{H}$ and $k_{D}$ (dimensionless parameter) values as obtained by the fit in the dilute regime at $T=30{ }^{\circ} \mathrm{C}$. In the case of the PMMA samples only a few $M_{w}$ values are reported. More extensive results on PMMA solutions can be found in Ref. 18 .

\begin{tabular}{crrrrrr}
\hline \hline Sample & \multicolumn{1}{c}{$M_{w}$} & $N$ & $R_{H}(\AA) \pm 5 \%$ & $k_{D} \pm 5 \%$ & $W^{*}$ & $C_{e}$ \\
\hline PMMA & 75000 & 750 & 58 & 4.1 & 0.18 & 0.22 \\
$\left(M_{e}=10000\right)$ & 120000 & 1200 & 79 & 3.9 & 0.12 & 0.15 \\
& 350000 & 3500 & 140 & 14.7 & 0.06 & 0.07 \\
& 800000 & 8000 & 217 & 21.4 & 0.04 & 0.04 \\
PEMA & 142000 & 1246 & 89 & 15.0 & 0.10 & 0.12 \\
$\left(M_{e}=8590\right)$ & 340000 & 2980 & 168 & 35.3 & 0.04 & 0.06 \\
& 850000 & 7456 & 276 & 55.2 & 0.02 & 0.03 \\
PBMA & 200000 & 1408 & 111 & 15.3 & 0.09 & 0.12 \\
$\left(M_{e}=12200\right)$ & 337000 & 2373 & 127 & 12.3 & 0.08 & 0.08 \\
& 800000 & 5634 & 208 & 26.3 & 0.045 & 0.04 \\
\hline \hline
\end{tabular}

allowed for a better determination of the background (a lower discrepancy between the measured and calculated baseline) we preferred the filtration method.

The investigated concentration range depends on the molecular weight because of the high viscosity of the samples. We collected data on the semidilute solutions usually for 4 or 5 days, repeating the same measurements for different delay times and scattering angles. The results were reproducible providing no evidence of aging effects.

QELS measurements were performed by means of a Photon Correlation Spectroscopy (PCS) technique, using a standard scattering apparatus, described in detail elsewhere. ${ }^{18,29,30}$ This technique allows one to investigate molecular motions on a time scale ranging from fractions of $\mu$ s to a few seconds; so it is suitable for the investigation of the slow modes. We also used a dual detector system operating in a cross-correlation mode in order to collect data also at fractions of $\mu$ s, without any distortion due to electronic noise. As an exciting source, the $4880 \AA$ vertically polarized line of a unimode $\mathrm{Ar}^{+}$laser was used. Samples were mounted in a thermostat with a temperature control better than $\pm 0.02{ }^{\circ} \mathrm{C}$ and the temperature was $303 \mathrm{~K}$.

QENS spectra were collected using the time-of-flight spectrometer FOCUS at the continuous neutron spallation source SINQ at the Paul Scherrer Institute $(\mathrm{CH})$, covering a momentum transfer domain from 0.28 to $1.93 \AA^{-1}$ and an energy transfer $E_{0}-E_{s}=\hbar \omega\left(E_{0}\right.$ and $E_{s}$ are the incident and scattered neutron energy, respectively) in the range between -3 and $1 \mathrm{meV}$.

We used an incoming neutron energy of $2.7 \mathrm{meV}$ providing an energy resolution of $\Gamma=60 \mu \mathrm{eV}$ [Full-Width at Half-Maximum (FWHM)] which was determined using a standard vanadium plate as a reference. The samples were contained in aluminum cells to obtain liquid samples in the form of $1 \mathrm{~mm}$ thick slabs. The thickness was chosen, according to the estimated transmission values, in order to obtain a good scattering signal combined with a minimized multiple scattering. The temperature during the experiment was $T$ $=303 \mathrm{~K}$.

An experimental run of the deuterated acetone showed that, when compared with the solution spectra, the solvent contribution can be neglected because of the low scattered intensity. 
Partially deuterated samples (namely with the deuterated backbone) were polymerized by Professor G. Maschio and Dr. G. De Domenico, working at the University of Messina, from selectively deuterated monomers furnished by the Aldrich Chemical Company. The comparison with the fully hydrogenated chains having the same polymerization degree allowed us to separate the contribution of the side group in the ester chain, and to characterize the complex dynamics of the polymer chains at the same temperature and different concentration values.

The raw spectra were corrected for background and detector efficiency and normalized using the FOCUS data analysis package NATHAN.

\section{DATA ANALYSIS}

It is well known that the PCS technique $e^{31,32}$ allows one to measure the correlation function of the scattered intensity $I_{S}(Q, t)$ :

$G_{2}(Q, t)=\left\langle I_{S}(Q, 0) I_{S}(Q, t)\right\rangle$.

If the scattered field obeys a Gaussian statistics, the Siegert's relationship can be applied:

$$
G_{2}(Q, t)=\langle I(Q)\rangle^{2}\left[1+\alpha\left|G_{1}(Q, t)\right|^{2}\right],
$$

where $\alpha$ is a constant which depends on the experimental set-up and $G_{1}(Q, t)$ is the field autocorrelation function. In the time domain typical for a light scattering experiment $\left(\geqslant 10^{-6} \mathrm{~s}\right)$, that is, for times between the characteristic viscous flow relaxation and the diffusive relaxation over a scale as great as the particle dimension, hydrodynamic interactions can be considered as instantaneous and direct interactions do not affect particle configuration. ${ }^{33}$

For diffusing monodisperse spherical scatterers, the intensity-intensity correlation function takes a simple exponential form: $G_{2}(t)=\langle I(Q)\rangle^{2}\left[1+\alpha \exp \left(-2 D_{c} Q^{2} t\right)\right]$, with $D_{c}$ the collective diffusion coefficient. The latter is defined by the generalized Stokes-Einstein relation $D_{c}$ $=(\partial \Pi / \partial c)_{T}[1 / \zeta(c)](1-V c)$, with $(\partial c / \partial \Pi)_{T} \propto[S(0)]^{-1}$ osmotic compressibility, $\zeta$ the frictional coefficient, $V$ the molecule partial specific volume and $c$ the concentration. 6,34 The concentration dependence of $D_{c}$ gives information about intermolecular interactions. In the dilute regime, in fact, the first order virial expansion can be applied:

$$
D_{c}=D_{0}\left(1+k_{D} c\right) \text {. }
$$

The sign and magnitude of the slope, $k_{D}$, are related to the frictional and direct interactions, by ${ }^{35}$

$$
k_{D}=2 A_{2} M-B_{\zeta}-2 V,
$$

with $B_{\zeta}$ and $A_{2}$ the second virial coefficients of the frictional interactions and of the osmotic compressibility, respectively.

In the very diluted limit, the theory of Brownian motion relates the measured $D$ to the self-diffusion coefficient $D_{0}$, the latter being connected with the hydrodynamic radius, $R_{H}$, of the particles by the Einstein-Stokes (ES) relation:

$$
D_{0}=\frac{K_{\mathrm{B}} T}{6 \pi \eta R_{H}},
$$

where $\eta$ is the viscosity coefficient of the continuous medium. $^{31}$

As a matter of fact, especially at a high molecular weight, the $G_{1}(t)$ of polymer solutions consists of a relaxation time distribution. Consequently, the standard cumulant analysis can be used. A fit using the Kohlrausch-WilliamsWatts (KWW) function ${ }^{36,37}$ is frequently employed as well:

$$
G_{1}(t)=A \exp \left\lfloor-\left(\frac{t}{\tau_{\mathrm{KWW}}}\right)^{\beta_{\mathrm{KWW}}}\right\rfloor,
$$

where $\beta_{\mathrm{KWW}}$, which ranges from 0 to 1 , is the shape parameter measuring the broadening of the relaxation time distribution. The average diffusive relaxation time is related to $\tau_{\mathrm{KWW}}$ by $\langle\tau\rangle=\left(\tau_{\mathrm{KWW}} / \beta_{\mathrm{KWW}}\right) \Gamma\left(1 / \beta_{\mathrm{KWW}}\right)$, with $\Gamma(x)$ the gamma function. In the dilute region the mean relaxation rates were calculated using both the cumulant expansion and the KWW function, finding the same results, within the experimental error (the shape parameter of the KWW has been found to be $0.9<\beta_{\mathrm{KWW}}<1$ in the dilute regime).

Above the overlap concentration value $c^{*}$ $=3 M\left(N_{\mathrm{A}} 4 \pi R_{H}^{3}\right)^{-1}$ (where $M$ is the molecular weight and $N_{\mathrm{A}}$ is the Avogadro number), in some solutions the evidence of the entanglement effect occurs in the presence of two distinct contributions in the intensity autocorrelation function. In the early De Gennes and Brochard theoretical description they showed ${ }^{9,16}$ that in the semidilute regime the field autocorrelation function takes the form

$$
G_{1}(Q, t)=A_{1} \exp \left(-\Gamma_{1} t\right)+A_{2} \exp \left(-\Gamma_{2} t\right)
$$

where $\Gamma_{1}=D_{g} Q^{2}$ and $\Gamma_{2}=D_{c} /\left(D_{g} \tau_{R}\right)$; with $D_{c}$ the collective diffusion coefficient (already defined), $D_{g}$ the diffusion coefficient of the transient gel and $\tau_{R}$ the characteristic lifetime of the network contact points formed by the entangled chains; the latter separates elastic and viscous regimes and depends strongly on the polymer molecular weight. Relation (7) holds, providing that the viscoelastic response (that is, the longitudinal elastic modulus) is characterized by a single exponential decay with $\tau_{R}$ as relaxation time. As a matter of fact $\tau_{R}$ have a very broad distribution and this occurrence has been taken into account in the data analysis of the correlation functions using KWW functions instead of simple exponentials [see Eq. (12)].

The faster relaxation mode, $\Gamma_{1}$, is controlled by the equilibration between osmotic pressure and elastic stress and reflects the relaxation $\left(Q^{2}\right.$-dependent) of the cooperative motions of the polymer chains forming a sort of network; the slower mode, $\Gamma_{2}$, is a $Q$-independent structural relaxation of the network itself and is related to the viscoelasticity of the entangled polymer chains.

QELS measures relaxation processes occurring on a mesoscopic space scale, involving a great number of molecules (or monomers in the present case); on the other hand, QENS probes the dynamical aspects of the system on a smaller space-time scale, allowing also a selective study of the processes involved by means of the isotopic substitution. 
The correlation function formalism ${ }^{38,39}$ provides a powerful and unifying framework for describing the scattering of neutrons from nuclei. In particular, the dynamic structure factor,

$$
\begin{aligned}
& S(\vec{Q}, \omega) \\
& =\frac{1}{2 \pi} \frac{1}{N} \int_{-\infty}^{+\infty}\left[\sum_{i, j} b_{i} b_{j}\left\langle\exp i \vec{Q} \cdot\left(\vec{r}_{i}(t)-\vec{r}_{j}(0)\right)\right\rangle\right] \\
& \quad \times \exp (-i \omega t) d t,
\end{aligned}
$$

which is the sum of both the coherent and the incoherent terms,

$$
\begin{aligned}
S^{\mathrm{coh}}(\vec{Q}, \omega) & \\
= & \frac{1}{2 \pi} \frac{1}{N} \int_{-\infty}^{+\infty}\left|\sum_{i, j} b_{i}^{\mathrm{coh}} b_{j}^{\mathrm{coh}}\left\langle\exp i \vec{Q} \cdot\left(\vec{r}_{i}(t)-\vec{r}_{j}(0)\right)\right\rangle\right| \\
& \quad \times \exp (-i \omega t) d t
\end{aligned}
$$

and

$$
\begin{aligned}
S^{\text {incoh }}(\vec{Q}, \omega) & \\
= & \frac{1}{2 \pi} \frac{1}{N} \int_{-\infty}^{+\infty}\left|\sum_{i}\left(b_{i}^{\text {incoh }}\right)^{2}\left\langle\exp i \vec{Q} \cdot\left(\vec{r}_{i}(t)-\vec{r}_{i}(0)\right)\right\rangle\right| \\
& \quad \times \exp (-i \omega t) d t
\end{aligned}
$$

reflects collective and individual atomic motions.

The differential scattering cross section in principle contains the superposition and interaction of a large number of vibrational, rotational and diffusive modes; for complex systems this implies that the explicit evaluation of $S(\vec{Q}, \omega)$ is very difficult or impossible. However, when the ratio of protons to other nuclei is close to unity, as in the present case, it is mainly the hydrogen atoms of the chains that contribute, and the incoherent spectrum is dominant in the structure factor. The self-dynamic of a hydrogen atom can always be decomposed into a translational contribution, a contribution arising from the motion relative to the center of mass and a contribution due to vibrations. This occurrence leads to the widely used simplification consisting in the decoupling of the different kinds of motions, which are supposed to be uncorrelated. On the basis of this hypothesis, the dynamic structure factor takes the form ${ }^{40}$

$$
S_{\text {inc }}(Q, \omega)=S^{\text {trans }}(Q, \omega) \otimes S^{\text {rot }}(Q, \omega) \otimes S^{\text {vib }}(Q, \omega) .
$$

If the vibrational frequencies are sufficiently high, the expansion of the scattering law for vibrations in powers of $\hbar \omega$, in the quasi-elastic region, gives a Debye-Waller factor contribution, $\exp \left(-Q^{2}\left\langle u^{2}\right\rangle\right),\left\langle u^{2}\right\rangle$ being the mean-square vibration amplitude. The Debye-Waller factor reflects the decrease of the total quasi-elastic intensity with increasing $Q$.

As a matter of fact, whereas the decoupling of vibrational dynamics is usually effective, rotational and translational motions can be so strongly correlated that, even if the decoupling approximation is a very useful working hypothesis, some caution in the interpretation of the data is necessary.

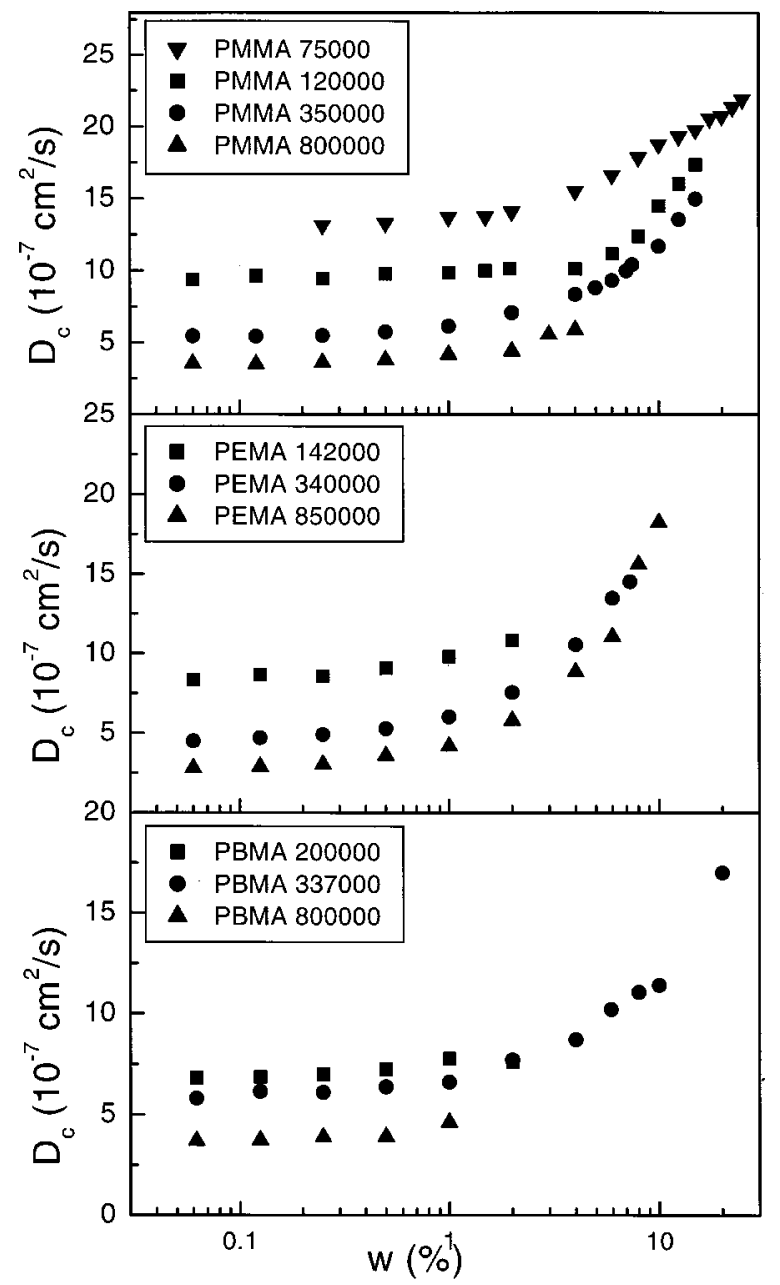

FIG. 1. Collective diffusion coefficient as a function of weight fraction for the different polymers at some molecular weight values. The dilute regime of PMMA was extensively studied in Ref. 18.

The fit procedure was performed according to $I(Q, \omega)$ $=S(Q, \omega) \otimes \operatorname{Res}(Q, \omega)+$ const, where $\operatorname{Res}(Q, \omega)$ is the resolution function as determined by the standard vanadium plate.

\section{RESULTS AND DISCUSSION}

In the present study we extend the findings already obtained regarding PMMA/acetone solutions ${ }^{18,41}$ for the other two kinds of polymers and also support them by the QENS results. Figure 1 reports the collective diffusion coefficient evolution versus the weight fraction as obtained by the autocorrelation functions for all the polymers at some molecular weight values, as examples. First, the conformational properties were evaluated in the dilute regime, deriving the size and the interaction character between the chains according to the relations (3) and (5). The obtained values are reported in Table I. Since all the three polymers obey the scaling law, $D_{0}=a M_{w}^{-\nu}$, with the scaling exponent varying from 0.57 for PMMA, to 0.55 for PEMA and 0.58 for PBMA, the solvent quality is good for all the three cases.

In the case of PMMA-acetone solutions, we showed that, for molecular weight values ranging from $75000 \mathrm{Da}$ to $800000 \mathrm{Da}$, the appearance of the slow modes ${ }^{18}$ is related to 


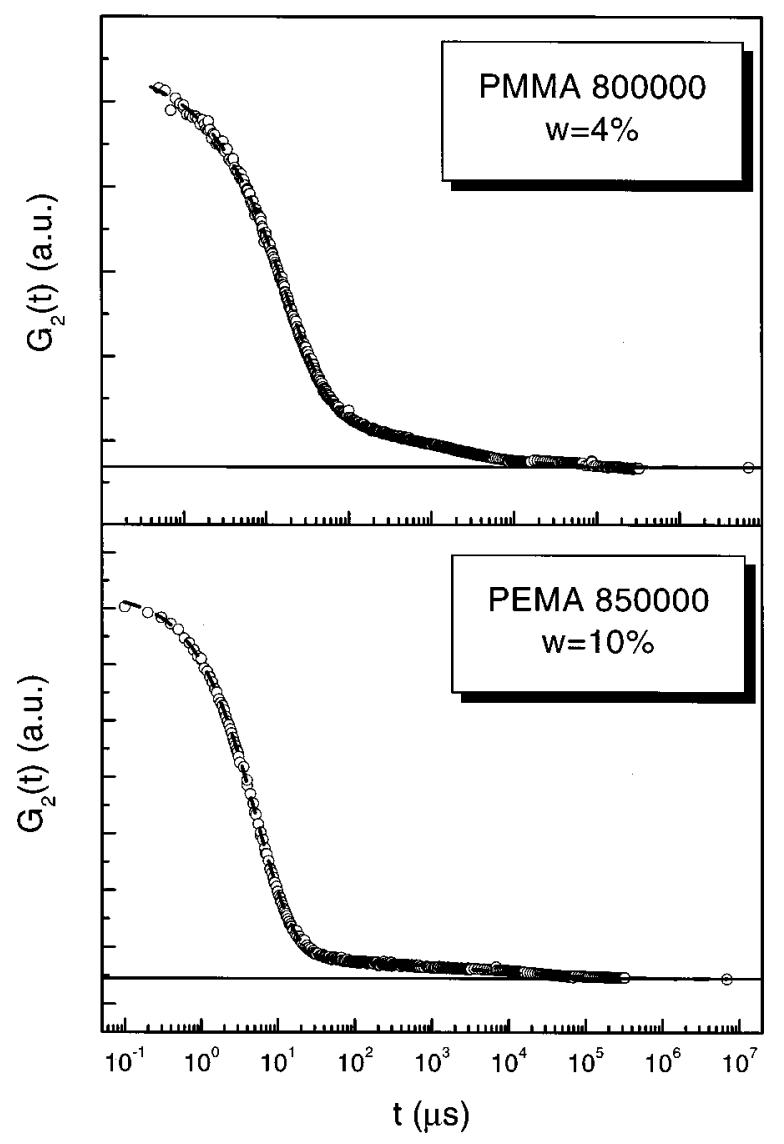

FIG. 2. Two examples of the intensity autocorrelation functions for PMMA and PEMA in the semidilute regime.

the overlap concentration, $w^{*}$; as concentration increases, the relative amplitude of these relaxation processes increases too. In Fig. 2, for example, the correlation function of PMMA 800000 at $w=4 \%$ is plotted. This function clearly shows two contributions and has been fitted using the following relation:

$$
\begin{aligned}
G^{(2)}(t)= & \left\{A_{1} \exp \left[-\left(t / \tau_{1}\right)^{\beta_{1}}\right]+A_{2} \exp \left[-\left(t / \tau_{2}\right)^{\beta_{2}}\right]\right\}^{2} \\
& + \text { BKG. }
\end{aligned}
$$

Equation (12) is analogous to Eq. (7), but here the two simple exponential decays have been replaced by Kohlrausch-Williams-Watts functions to account for the broad distribution of the slow relaxation process. However, whereas $\beta_{2}$ is relatively low $(0.5-0.7), \beta_{1}$ is close to unity for all the samples.

The results obtained for PEMA with a molecular weight of $850000 \mathrm{Da}$ (the polymerization degree is $N=7456$ ), despite the concentration being well above the overlap value, show that the slow modes are hardly detectable (Fig. 2). The structural relaxation contribution appears only for very long chains; in fact, for PEMA with a molecular weight of 340000 $\mathrm{Da}$ (corresponding to a polymerization degree of 4500), the slow modes do not appear.

Concerning PBMA, which has the longest side chain, we investigated concentrated solutions (above $w=30 \%$ ) with a
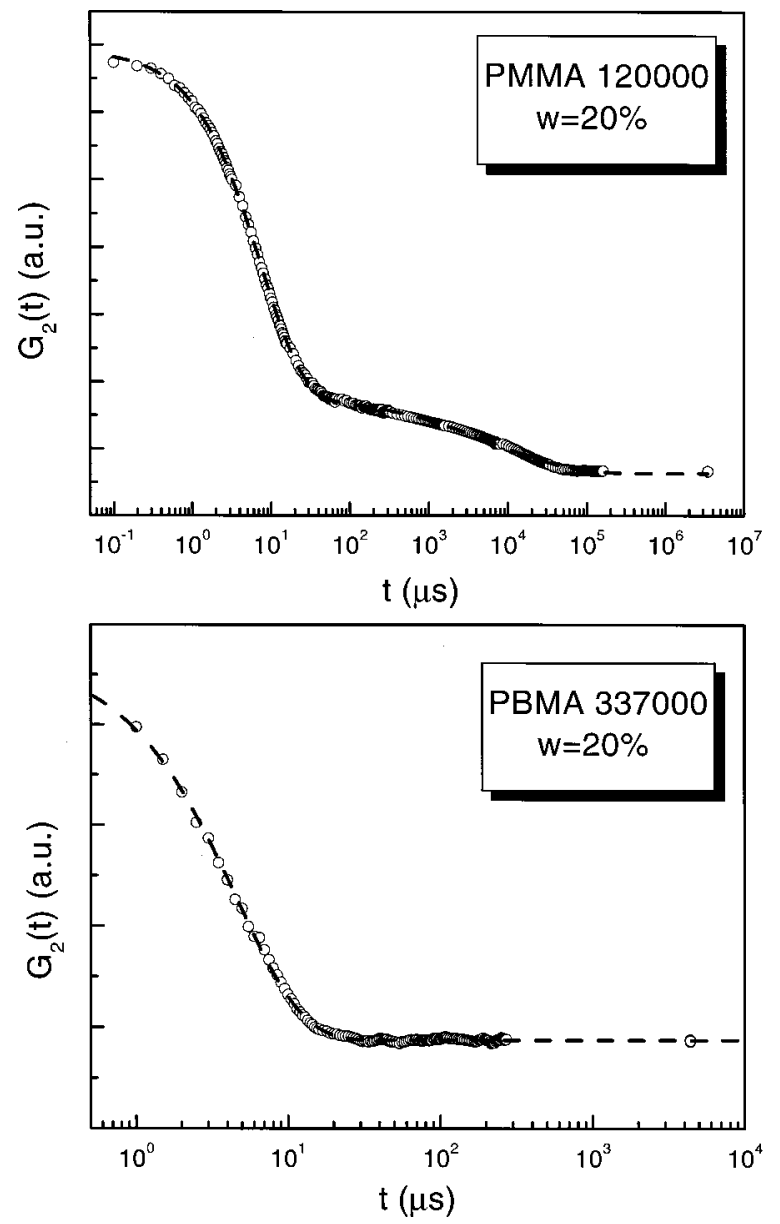

FIG. 3. A comparison with the case of PBMA in which the slow modes do not appear at a high concentration.

molecular weight of $337000(N=2373)$. Despite the high concentration, we observe no presence of slow modes (see Fig. 3).

These results can be rationalized by introducing the entanglement concentration, defined as the concentration at which polymers become entangled in a good solvent, or the entanglement molecular weight at a certain concentration value. Such values have been calculated according to the following formulas ${ }^{42,43}$ (see Table I): (i) $c_{e}=\left(M / M_{e}\right)^{-0.76}$; (ii) $M_{e, w}=M_{e} / w, M$ and $M_{e}{ }^{44,45}$ being the molecular weight of the polymer and the entanglement molecular weight in the bulk, respectively. In fact, it has been $\operatorname{seen}^{43}$ that at $w^{*}$ polymer chains could not be in the entangled state. However, in the present case, $w^{*}$ and $c_{e}$ do not differ very much and the solutions investigated in the semidilute regime do reach the entangled state. According to these calculations, at concentrations close to or higher than $c_{e}$, the slow relaxation mode should be detectable for almost all the samples. Evaluating the entanglement degree in the solutions through $M_{e, w}$, the calculation at $w=0.1$ (as an example) yields the following results: $M_{e, 0.1}(\mathrm{PMMA})=100000, \quad M_{e, 0.1}(\mathrm{PEMA})=85900$, $M_{e, 0.1}(\mathrm{PBMA})=122000$. Therefore, both methods, independently, indicate that the polymer chains in the investigated solutions are entangled.

The experimental results, however, show that the slow modes are present in PMMA and PEMA solutions, but no 


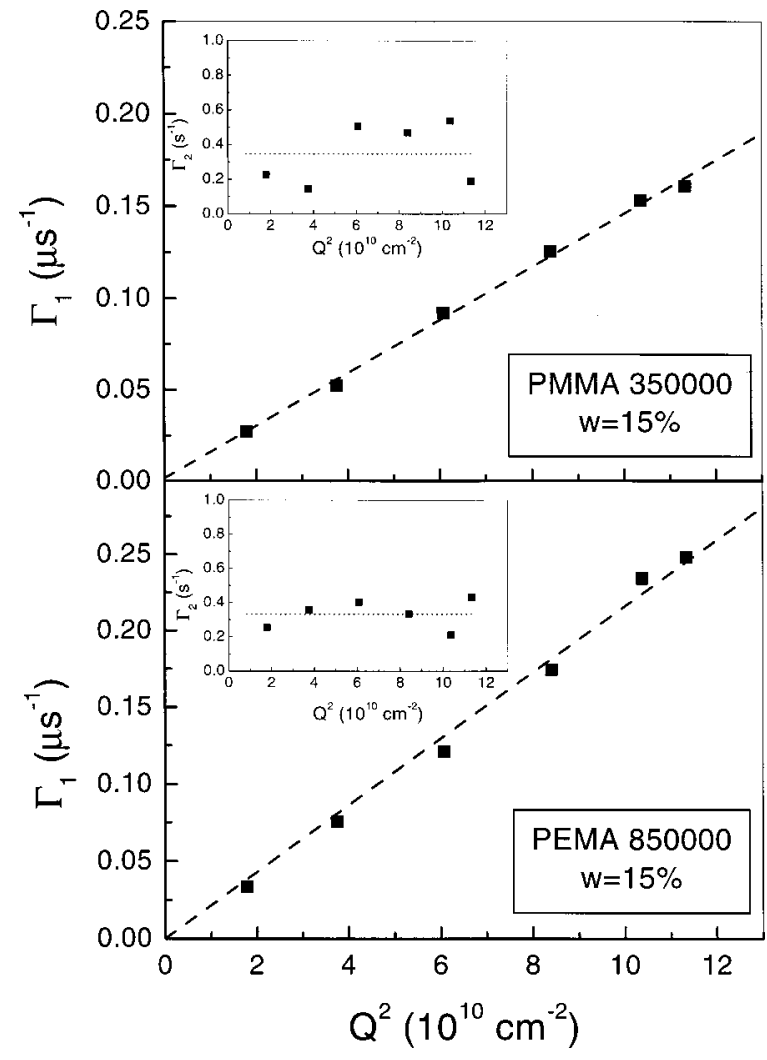

FIG. 4. Fast and slow decay rates for PMMA and PEMA solutions.

evidence of them is found for PBMA solutions. Moreover, the amplitude of the slow component is very different in the three polymer solutions, being much more significant in PMMA. We also measured the value of the Wang's $\beta$ parameter ${ }^{10,15}$ for all the investigated polymer solutions. The data indicate that $\beta$ is nonzero at all concentration values, decreasing only slightly (about 10\%) from PMMA to PBMA solutions; in other words polymer and solvent components do not have the same partial specific volumes. This suggests that the appearance of the slow mode is not directly related only to the value of $\beta$.

In Fig. 4, the $Q$-dependence of the fast and slow decay rates of the autocorrelation functions is reported for PMMA and PEMA solutions at a concentration above the overlap value. The results obtained show that the fast relaxation mode is diffusive; it is due to the collective diffusion of blobs. The slow one is $Q$-independent and can be interpreted on the basis of the structural rearrangements of the polymer transient network.

The comparative investigation of these homologous polymers seems to show that the slow modes are connected to the entanglement phenomena and that the main chain length drives their appearance. On the other hand, the fact that in PMMA solutions these modes have significant amplitude also at concentration near $c_{e}$ and for lower molecular weight than the other investigated polymers suggests the possibility that for PMMA chains the disentanglement phenomenon is more hindered.

In order to focus attention on the chain segmental mobility in the semidilute regime from a microscopic point of view, the investigation was carried out by QENS. The fully

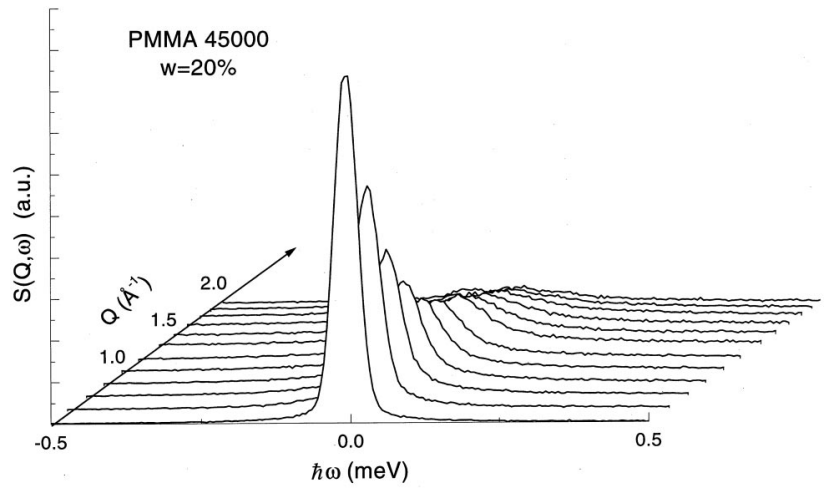

FIG. 5. Quasi-elastic spectra from $Q=0.28$ to $1.93 \AA^{-1}$ relative to the backbone dynamics for PMMA 45000.

hydrogenated and partially deuterated chains allow us to distinguish the side unit's motions and its effects on the main chain dynamics. Figure 5 shows the spectra obtained by subtracting the hydrogenated from the deuterated spectra at different $Q$ values for PMMA with $M_{w}=45000$. The dynamic, reproduced by the dynamic structure factor, is described by the following fit law: $:^{40,46,47}$

$$
\begin{aligned}
S(Q, \omega)= & A(Q)\left\lfloor F(Q) \frac{1}{\pi} \frac{\Gamma_{1}(Q)}{\Gamma_{1}^{2}(Q)+\omega^{2}}\right. \\
& \left.+(1-F(Q)) \frac{1}{\pi} \frac{\Gamma_{2}(Q)}{\Gamma_{2}^{2}(Q)+\omega^{2}}\right\rfloor .
\end{aligned}
$$

In particular, $F(Q)$ is the Elastic Incoherent Structure Factor (EISF) factor, which represents an average of the diffraction pattern produced on the neutron wave by all possible configurations of all the scattering centers. In the present case, only the moving incoherent scattering center (a proton) is considered and $F(Q)$ is the diffraction pattern on all its possible final positions, namely the Fourier transform of the "trajectory" of the proton. The two Lorentzians, whose HWHM are $\Gamma_{1}$ and $\Gamma_{2}$, respectively, take into account translational and rotational motions.

As far as the rotational contribution is concerned, it can be shown that, quite generally, the (incoherent) scattering law of any rotational model can be written as ${ }^{40}$

$S_{\mathrm{rot}}(Q, \omega)=F(Q) \delta(\omega)+\frac{1}{\pi} \sum_{l=1}^{\infty}$ broadened terms,

where the broadened terms are usually represented by Lorentzian contributions.

The contribution due to the methyl group rotation is so fast that it can be considered indistinguishable from the background.

The relation (13) is the result of the convolution between a Lorentzian representing the translational contribution and $S_{\text {rot }}(Q, \omega)$ for which we considered only the delta function and the first term of the series in (14). The good fit quality 


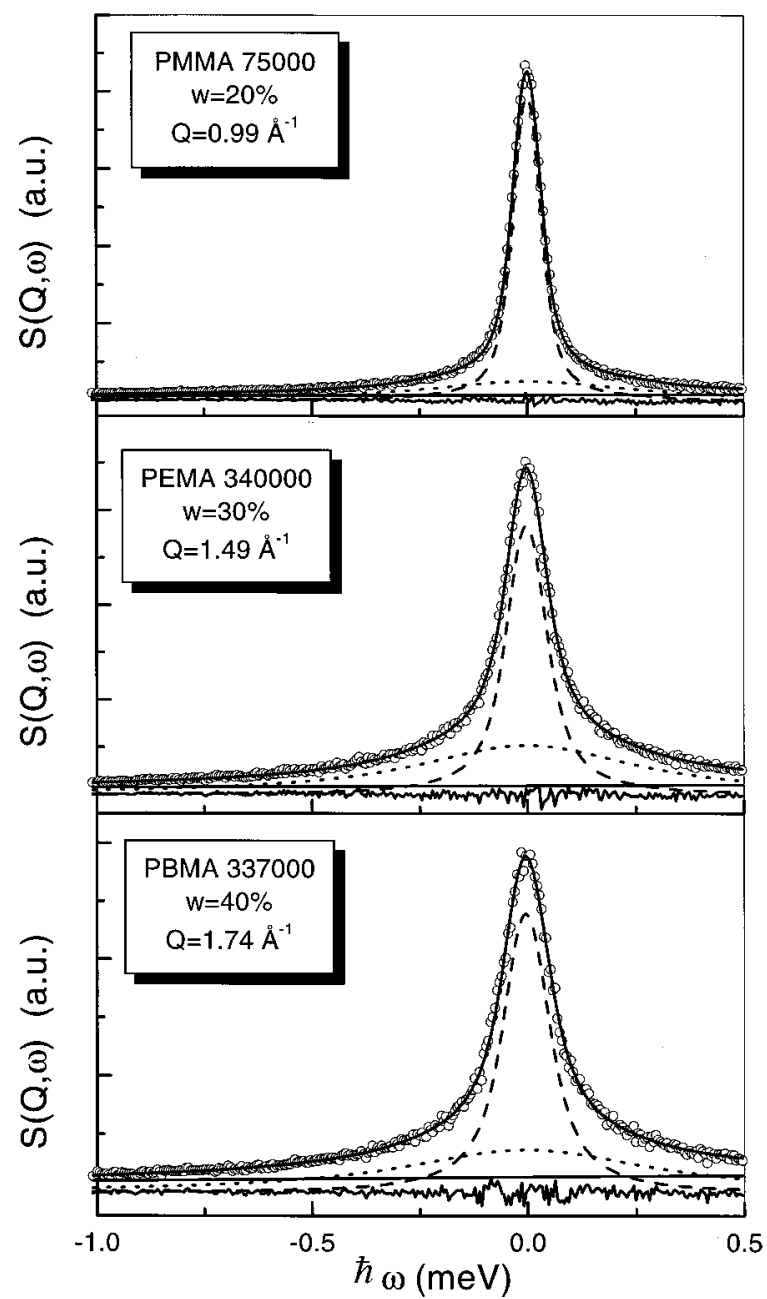

FIG. 6. Experimental spectra of the polymer solutions. The continuous line is the fit result obtained adopting the relation (13); the dashed narrower Lorentzian and the dotted broader one refer to the transnational and rotational motion of the segments, respectively. The background and the residuals are shown as well.

justifies a posteriori such an approximation, as can be seen from an inspection of Fig. 6, where we report, for different spectra, the fit result together with all the components as obtained adopting the relation (13).

Figure 7 shows the Half-Width at Half Maximum (HWHM), $\Gamma_{1}$, of the first Lorentzian as a function of $Q^{2}$ for two concentration values of a PMMA 45000 solution. The linear behavior suggests a diffusive dynamic of the segments belonging to the main chain. In fact, it is known ${ }^{48}$ that for $Q R_{g} \ll 1$ the decay rate is $Q^{2}$-dependent; for $Q R_{g} \gg 1$ and $Q a \ll 1, a$ being the monomer dimension, the probe is sensitive to the internal dynamics of the polymer $\left(\Gamma \sim Q^{3}\right)$. Finally for $Q a \geqslant 1$ the dynamic is diffusive again. In the present case $Q a \geqslant 1$, except for the first two $Q$ values, and according to the model just described, the dynamic of the slower component reproduces the diffusive motion of the chain segments. The comparison between the $\Gamma$ values at different concentrations shows that the dynamic is evidently more hindered because of the topological constraint obstructing the chain mobility.

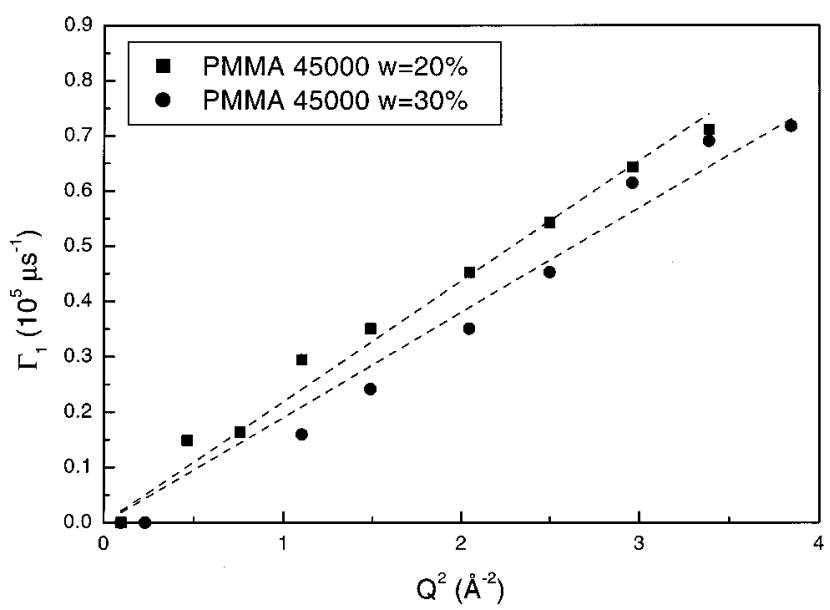

FIG. 7. Half-width at half-maximum evolution of the quasi-elastic line of PMMA 45000 at a different concentration.

As far as the second Lorentzian contribution is concerned, $\Gamma_{2}$ is $Q$-independent, suggesting that reorientation of the segments are allowed on a time scale of $\sim 3$ ps.

In Fig. 8 the diffusive dynamics of the three polymers

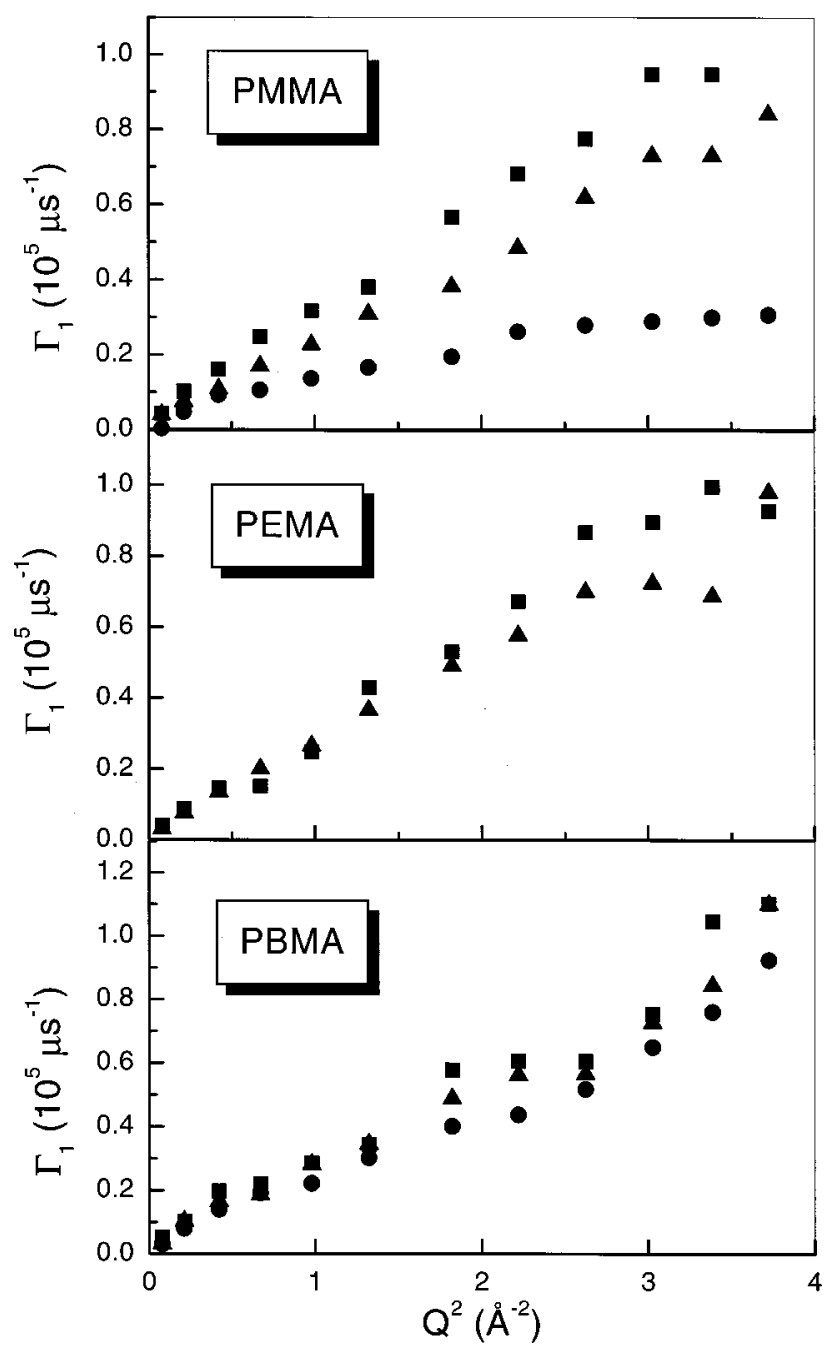

FIG. 8. Half-width at half-maximum for PMMA 75000, PEMA 340000 and PBMA 337000 solutions at different concentration values: (ם) $w=20 \%$; (ム) $w=30 \% ;(\bullet) ~ w=40 \%$. 


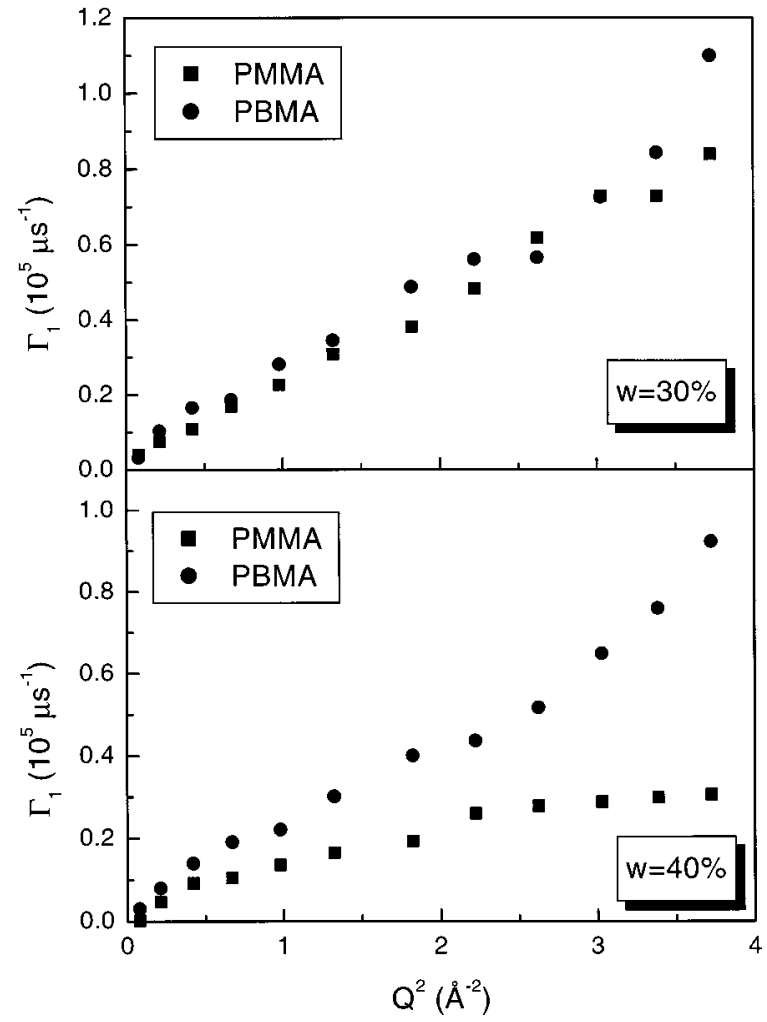

FIG. 9. A comparison between the half-width at half-maximum evolutions of PMMA 75000 and PBMA 340000 solutions at the same concentration.

are reported. The main feature is a clearly stronger concentration-dependence of the segmental diffusion of PMMA chains than that of the other polymers. The comparison between the different polymers at the same concentration value, in our opinion, is reasonable because the volume fraction occupied by the polymer, estimated in the approximation of ideal mixing from density measurements, can be considered roughly the same at the same weight fraction.

In order to check the existence of glass transitionconnected effects in the spectra, we made an estimate of the glass transition temperature of the solution, starting from that of the polymer, through the following relation: ${ }^{45}$

$$
T_{g}=\frac{T_{g p}+\left(k T_{g s}-T_{g p}\right) \Phi_{s}}{1+(k-1) \Phi_{s}},
$$

where $T_{g p}, T_{g s}, \Phi_{s}$ are the glass transition temperature of the polymer, the glass transition temperature of the solvent, and the volume fraction of the solvent, and $k=2.5$ is a parameter depending on the polymer.

All the polymer solutions were investigated well above the glass transition temperature, for example, at $w=40 \% T_{g}$ $($ PMMA 120000$)=239 \mathrm{~K}, T_{g}($ PEMA 850000$)=231 \mathrm{~K}$, and $T_{g}($ PBMA 337000$)=223 \mathrm{~K}$. Moreover, the $T_{g}$ values of the solutions between $w=20 \%$ and $w=40 \%$ vary in the same range of about 15 degrees.

Figure 9, therefore, shows that the higher the concentration, the more marked are the differences in the diffusive behaviors of the three polymers. But what is particularly striking is that the PMMA segmental dynamics appear to be slower than those of the other polymers, especially at $w$ $=40 \%$, even if the main chain length and the side unit size are smaller. In Table II we report the diffusion coefficient
TABLE II. Diffusion coefficient values obtained by the $Q^{2}$-dependence of the quasi-elastic linewidth of the neutron spectra.

\begin{tabular}{lccccc}
\hline \hline & & \multicolumn{4}{c}{$D\left(10^{-6} \mathrm{~cm}^{2} / \mathrm{s}\right)$} \\
\cline { 3 - 6 } Sample & $M_{w}$ & $10 \%$ & $20 \%$ & $30 \%$ & $40 \%$ \\
\hline \multirow{2}{*}{ PMMA } & 45000 & - & 2.3 & 2.0 & - \\
& 75000 & - & 2.7 & 2.1 & 0.9 \\
\multirow{2}{*}{ PEMA } & 340000 & - & 2.7 & 2.3 & - \\
& 850000 & - & 1.8 & - & - \\
PBMA & 337000 & - & 2.9 & 2.6 & 2.1 \\
& 800000 & 3.2 & - & - & - \\
\hline \hline
\end{tabular}

values, related to the segmental motion of the overlapped chains, as obtained from the slope of $\Gamma$ versus $Q^{2}$.

In Fig. 10 the $F(Q)$ factor is reported. In all the cases, $F(Q)$ does not reach the zero value at high $Q$. This can be due to a certain part of the molecules being unable to reorientate because of both the connected structure of the polymer and entanglement phenomena. Taking into account the two different cases of PMMA and PBMA at two concentration values, it can be seen that, increasing concentration and decreasing the side unit length, the confined region of the motion becomes smaller.

Finally, from an inspection of the Debye-Waller factor [identified as $A(Q)$ in the relation (13)] of all the systems it is possible to extract a value of the root mean square dis-

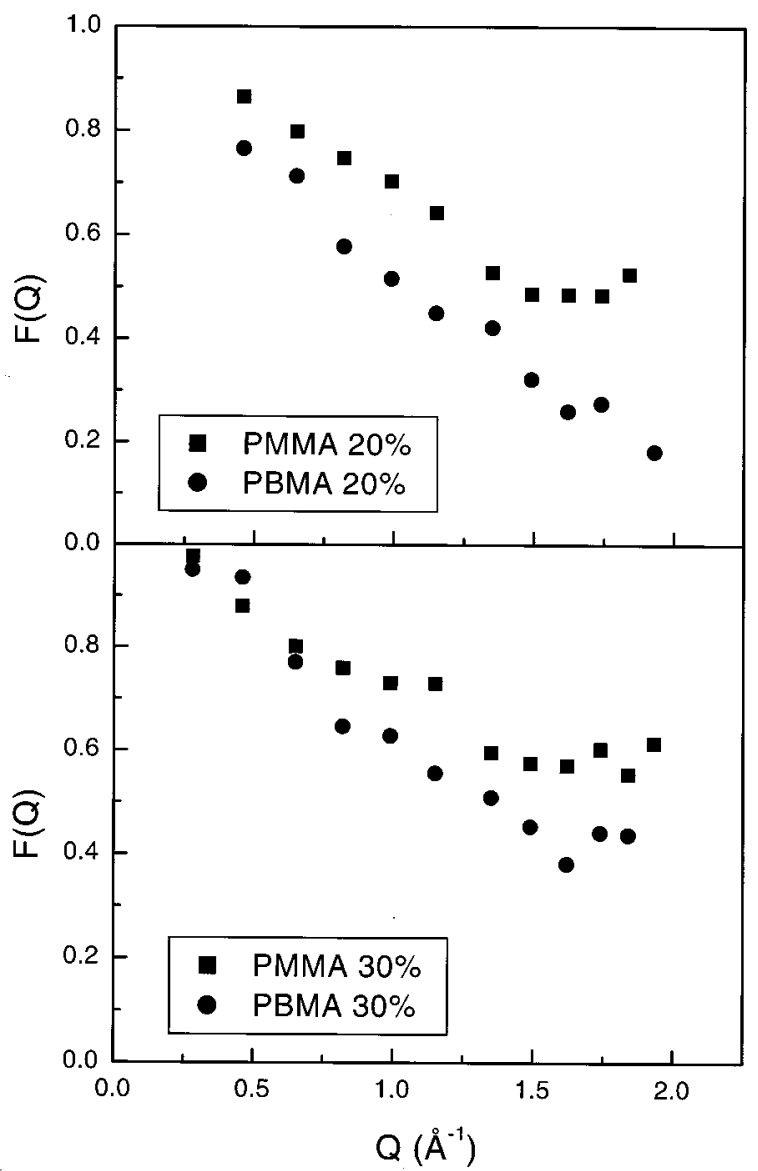

FIG. 10. EISF factor relative to the segmental dynamics in PMMA 75000 and PBMA 340000 solutions. 
placement: It is equal to $0.3 \AA$, independently from the structure of the polymer or concentration of the solutions.

In view of these results, the absence of the slow modes in the autocorrelation functions of PEMA at low molecular weight, and of PBMA, is likely connected with their higher segmental mobility compared with PMMA. This occurrence could be traced back to the obstruction of the chains' overlapping process because of the bigger monomer unit, and, consequently, to the reduction of the effective topological constraints and of the entanglement processes giving rise to the slow relaxation modes.

\section{CONCLUSIONS}

The occurrence of the slow modes, as shown in many investigations, and their $Q$-dependence seem to be a systemdependent phenomenon; this system dependence creates difficulty in providing an all-embracing explanation for their microscopical origin. In the study reported in the present paper on polyalkylmethacrylate solutions we aim to show how the structural and conformational properties affect the chain dynamics in the entangled solutions. The results show that homologous polymers having a different side unit length display considerably different dynamical properties in the semidilute regime. In fact, at concentration values higher than $c_{e}$ the slow modes are experimentally detectable in PMMA and PEMA solutions, but they do not appear in the PBMA solutions. The results from QENS, moreover, suggest a stronger concentration-dependence of the segmental diffusion of PMMA chains than that of the other polymers.

On the basis of these results it seems that longer side chains can give rise to larger excluded volume to the other segments, facilitating local segregation and obstructing the entanglements responsible for the slow relaxation modes. The overall view is an increased mobility of the segments with longer side units.

\section{ACKNOWLEDGMENTS}

The authors would like to thank Professor G. Maschio and Dr. G. De Domenico of the University of Messina (Dipartimento di Chimica Industriale) for having performed polymerization of partially deuterated samples and the Paul Scherrer Institute $(\mathrm{CH})$ for the beam time allocated on FOCUS.

${ }^{1}$ P. J. Flory, Statistical Mechanics of Chain Molecules (Interscience, New York, 1969).

${ }^{2}$ L. J. Fetters, D. J. Lohse, D. Richter, T. A. Witten, and A. Zirkel, Macromolecules 27, 4639 (1994).

${ }^{3}$ J. D. Ferry, Viscoelastic Properties of Polymers, 2nd ed. (Wiley, New York, 1970)

${ }^{4}$ P. Stepanek and W. Brown, Macromolecules 31, 1889 (1998).

${ }^{5}$ T. Jian, D. Vlassopoulos, G. Fytas, T. Pakula, and W. Brown, Colloid Polym. Sci. 274, 1033 (1996).

${ }^{6}$ W. Brown and T. Nicolai, in Dynamic Light Scattering: The Method and Some Applications (Clarendon, Oxford, UK, 1993), chap. 6.

${ }^{7}$ Y. Einaga and D. Karube, Polymer 40, 157 (1998).
${ }^{8}$ A. Rtfzl, L. Belkoura, and D. Woermann, Phys. Chem. Chem. Phys. 1, 1947 (1999).

${ }^{9}$ F. Brochard and P. G. de Gennes, Macromolecules 10, 1157 (1977).

${ }^{10}$ C. H. Wang, J. Chem. Phys. 95, 3788 (1991).

${ }^{11}$ M. Doi and A. Onuki, J. Phys. II 2, 1631 (1992).

${ }^{12}$ A. N. Semenov, Physica A 166, 263 (1990).

${ }^{13}$ C. H. Wang, in Dynamic Light Scattering: The Method and Some Applications (Clarendon, Oxford, UK, 1993), Chap. 7.

${ }^{14}$ A. Z. Ackasu, R. Klein, and C. H. Wang, Macromolecules 27, 2736 (1994).

${ }^{15}$ C. H. Wang, J. Chem. Phys. 102, 6537 (1995).

${ }^{16}$ M. Adam and M. Delsanti, Macromolecules 18, 1760 (1985).

${ }^{17}$ W. Brown and P. Stepanek, Macromolecules 26, 6884 (1993); C. H. Wang, Z. Sun, and Q. R. Huang, J. Chem. Phys. 105, 6052 (1996).

${ }^{18}$ A. Faraone, S. Magazù, G. Maisano, R. Ponterio, and V. Villari, Macromolecules 32, 1128 (1999).

${ }^{19}$ H. Wang, C. Jarnagin, and E. T. Samulski, Macromolecules 27, 4705 (1994)

${ }^{20}$ C. L. Hoffmann, H. T. Man, and G. G. Fuller, Acta Polym. 44, 39 (1993).

${ }^{21}$ H. Ohno, Y. Inoue, and P. Wang, Solid State Ionics 62, 257 (1993).

${ }^{22}$ W. Brown and P. Stepanek, J. Polym. Sci., Part B: Polym. Phys. 35, 1013 (1997)

${ }^{23}$ G. Fytas, A. Patkowski, G. Meier, and Th. Dorfmüller, J. Chem. Phys. 80, 2214 (1984).

${ }^{24}$ K. Schröter, R. Unger, S. Reissig, F. Garwe, S. Kahle, M. Beiner, and E. Donth, Macromolecules 31, 8966 (1998).

${ }^{25}$ F. Garwe, A. Schönhals, H. Lockwenz, M. Beiner, K. Schröter, and E. Donth, Macromolecules 29, 247 (1996).

${ }^{26}$ G. Meier, in Molecular Dynamics and Relaxation Phenomena in Glasses (Springer-Verlag, Berlin, 1987).

${ }^{27}$ J. Spevacek and M. Suchopárek, Macromolecules 30, 2178 (1997); N. Fazel, A. Brulet, and J.-M. Guenet, ibid. 27, 3836 (1994).

${ }^{28}$ A. Hwuang, G. D. Patterson, and J. R. Stevens, J. Polym. Sci., Part B: Polym. Phys. 34, 2291 (1996).

${ }^{29}$ A. Faraone, S. Magazù, G. Maisano, P. Migliardo, E. Tettamanti, and V. Villari, J. Chem. Phys. 110, 1801 (1999).

${ }^{30}$ S. Magazù, G. Maisano, P. Migliardo, and V. Villari, J. Chem. Phys. 111, 9086 (1999).

${ }^{31}$ B. J. Berne and R. Pecora, Dynamic Light Scattering with Application to Chemistry, Biology and Physics (Wiley, New York, 1976).

${ }^{32}$ H. Z. Cummins, in Photon Correlation and Light Beating Spectroscopy, edited by H. Z. Cummins and E. R. Pike (Plenum, New York, 1974).

${ }^{33}$ R. Klein and G. Nagele, Nuovo Cimento 16, 963 (1994).

${ }^{34}$ P. N. Pusey and R. J. A. Tough, in Dynamic Light Scattering, edited by R. Pecora (Plenum, New York, 1985), Chap. 4.

${ }^{35}$ K. Devanand and J. C. Selser, Macromolecules 24, 5943 (1991).

${ }^{36}$ R. Kohlraush, Ann. Phys. (Leipzig) 91, 179 (1954).

${ }^{37}$ G. Williams and D. C. Watts, Trans. Faraday Soc. 66, 80 (1970).

${ }^{38}$ M. Bee, Quasielastic Neutron Scattering, edited by A. Hilger (Bristol and Philadelphia, 1988).

${ }^{39} \mathrm{~F}$. Volino, in Microscopic Structure and Dynamics of Liquids, edited by J. Dupuy and A. J. Dianoux (Plenum, New York, 1978).

${ }^{40}$ F. Volino and A. J. Dianoux, Organic Liquids; Structures Dynamics and Chemical Properties (Wiley, New York, 1978).

${ }^{41}$ A. Faraone, S. Magazù, G. Maisano, G. Maschio, and V. Villari, Macromol. Chem. Phys. 200, 1134 (1999).

${ }^{42}$ M. Adam, D. Lairez, and E. Raspaud, Phys. Rev. Lett. 77, 3673 (1996).

${ }^{43}$ P. Stepanek and W. Brown, Macromolecules 31, 189 (1998).

${ }^{44}$ D. Porter, Group Interaction Modelling of Polymer Properties (Dekker, New York, 1995).

${ }^{45}$ D. W. Van Krevelen, Properties of Polymers (Elsevier, New York, 1990).

${ }^{46}$ A. Deriu, F. Cavatorta, D. Di Cola, and H. D. Middendorf, J. Phys. IV 3, 237 (1993).

${ }^{47}$ S. Magazù, V. Villari, G. Maisano, P. Migliardo, and M. T. F. Telling, J. Phys. Chem. 105, 1851 (2001).

${ }^{48}$ D. W. Schaefer and C. C. Han, in Dynamic Light Scattering, edited by R. Pecora (Plenum, New York, 1985), chap. 5. 